\title{
The Association between Stress and Sexual Dysfunctionality in Men and Women: A Systematic Review
}

\author{
Michael Galanakis ${ }^{1}$, Maria-Despoina Kallianta ${ }^{2}$, Chrysoula Katsira ${ }^{2}$, Dimitra Liakopoulou ${ }^{2}$, \\ P. George Chrousos ${ }^{2,3 *}$, Christina Darviri ${ }^{* *}$ \\ ${ }^{1}$ Panteion University of Social and Political Sciences, Athens, Greece \\ ${ }^{2}$ Postgraduate Course Science of Stress and Health Promotion, School of Medicine, University of Athens, \\ Athens, Greece \\ ${ }^{3}$ First Department of Pediatrics, Children's Hospital Aghia Sofia, School of Medicine, University of Athens, \\ Athens, Greece \\ Email: "cdarviri@yahoo.com
}

Received 30 September 2015; accepted 16 November 2015; published 19 November 2015

Copyright (C) 2015 by authors and Scientific Research Publishing Inc.

This work is licensed under the Creative Commons Attribution International License (CC BY). http://creativecommons.org/licenses/by/4.0/

\section{c) (i) Open Access}

\section{Abstract}

Purpose: Sexuality and sexual functionality are considered as an important factor of physical and psychological health. Over the years, problems with sexual functionality have been related to stress. A systematic review of the articles concerning sexual dysfunctionality is presented in this article, with emphasis in the past five years in order to highlight new aspects and implications. Method: The study was based on relevant articles from the PubMed and Wiley online library, using the combination of keywords stress and sexual dysfunctionality, while excluding articles prior to 2010. Results: The analysis shows that the phenomenon is complex and multifactorial. Variables such as depression, PTSD, lifestyle, gender, personality and positive coping play a crucial role. Research findings also indicate that sexual dysfunctionality is often misdiagnosed and not immediately taken care of. Conclusion: The severity of the pathology regarding sexual dysfunctionality calls for immediate planning and coping. Finally future research directions are discussed.

\section{Keywords}

Sexual Dysfunctionality, Stress, Gender, Systematic Review

\footnotetext{
${ }^{*}$ These authors contributed equally and shared last authorship.

" Corresponding author.
} 


\section{Introduction}

Sexuality is instinctive in human beings and sexual function is an important determinant of health and wellbeing. The prevalence of sexual dysfunction is high, although the risk factors have not been studied thoroughly yet (Shadman et al., 2014). Stress has always been correlated with sexual dysfunctions leading therapists to focus on stress as an explanation of their patients' sexual problems. In DSM-5 only three female dysfunctions and four male dysfunctions are listed, as opposed to five and six, respectively, in the DSM-IV (IsHak \& Tobia, 2013). Female hypoactive desire dysfunction and female arousal dysfunction were merged into a single syndrome called sexual interest/arousal disorder. Similarly, the formerly separate dyspareunia and vaginismus are now called genital pelvic pain/penetration disorder. Female orgasmic disorder remains in place. As for males, male hypoactive sexual desire disorder now has a separate entry. Male orgasmic disorder was changed to delayed ejaculation, the "male" adjective was dropped from erectile disorder, and premature ejaculation remained unchanged. Male dyspareunia or male sexual pain does not appear in the sexual dysfunctions chapter of the DSM-5. Two more significant alterations of DSM-5 are, firstly, the fact that for sexual dysfunctions to be diagnosed, they must last longer than 6 months and, secondly, that they have to cause significant distress. Stress, according to Lazarus \& Folkman (1984), is a cognitive appraisal, a process through which the person evaluates whether a particular encounter with the environment is relevant to his or her well-being and if so, in what ways. Furthermore, stress leads to the disruption of homeostasis and is followed by psychological, behavioral and physiological changes. The aim of this study is to systematically review the latest evidence base among stress, anxiety and sexual dysfunctions in men and women.

\section{Method}

The literature on this subject was searched through databases including PubMed and Wiley Online Library plus other electronic hand-searching. The eligibility criteria include Randomized Control Trials (RCTs), Cross sectional studies, Cohort Studies, Systematic Reviews, in adult men and women. Only free available full-texts dated from January 2010 until June 2015 were included. Non English publications were not included. The search was narrowed down to articles including both the words stress and sexual dysfunctionality at the same time.

Updated electronic search yielded 80 articles in total from PubMed $(n=66)$ and Wiley Online Library $(n=$ 14). 9 duplicate were removed. 71 unique citations of titles and abstracts were reviewed. However 56 citations were removed that did not meet inclusion criteria (non-relevant measurements, un-related topic, sexual dysfunction and stress not assessed as an outcome). Thus, 15 articles were included plus 1 that had been electronically hand-searched. Finally, there were a total number of 16 articles.

\section{Results}

Most of the studies were conducted separately on men and women, except for two. The smallest sample was $\mathrm{N}=$ 19 for women and $\mathrm{N}=439$ for men, and the largest sample was $\mathrm{N}=1241$ women and $\mathrm{N}=405,275$ men. The two mixed sample studies had a sample of $\mathrm{N}=198$ and $\mathrm{N}=4039$. The ages of the samples fluctuated from 18 to 62 years old. The samples were collected at therapeutical centers, universities or via advertisements in newspapers and internet.

The most common questionnaires used for the assessment of sexual dysfunctions on women were Female Sexual Function Index (FSFI), Index of Sexual Life (ISL) and the categorization of ICD9-CM code. The Subjective Response Scale and the Sexual Dysfunction Scale (SDS) were also used, while genital arousal was measured, using a vaginal photoplethysmograph and arousometer. For men ISL was used alongside with the International Index of Erectile Function and systemic evaluations by an andrologist, urology visits and medications. Also an Internet-assisted questionnaire developed by the PE Study Group of the Korean Andrological Society was used in one study.

Stress was measured using various methods, mostly using questionnaires and scales that show high validity. The most commonly used Scale was the Female Sexual Distress Scale-Revised (FSDS-R). Other assessment tools used were the Depression Anxiety Stress Scales (DASS-42), the Clinician-Administered PTSD Scale (CAPS) Symptoms of PTSD, the Sexual Satisfaction Scale for Women (SSS-W), the Hassles Scale, the Spielberger State-Trait Anxiety Index (STAI), the Beck Anxiety Inventory (BAI), the Life events Questionnaire and the Symptom Check List 90 revised (SCL-90-R) plus the Internet-assisted questionnaire developed by the PE 
Study Group of the Korean Andrological Society Personal Experiences Questionnaire. Other assessments were made through measurements of hormone levels that are correlated to stress such as follicle-stimulating hormone (FSH), luteinizing hormone (LH), testosterone (T), prolactin, and estradiol, salivary cortisol and DHEAS levels. Mental health diagnoses were identified using ICD-9-CM diagnoses corresponding to the Diagnostic and Statistical Manual of Mental Disorders, Fourth Edition classifications.

Psychological variables when evaluating women have a significant role for sexual dysfunction. Studies show that distress is strongly related with sexual dysfunction. Two of the studies that were conducted on young female students showed that stress, tiredness and disease interferes to sexual life and leads to sexual bother and to high risk of female sexual dysfunction (HRFSD) explaining that stress and depression are directly correlated and lead to female sexual dysfunction, such as orgasmic problems, creating a vicious cycle (Shindel et al., 2011; Shindel et al., 2013). Another study that confirms the correlation between stress, depression and sexual dysfunction focuses on increased pelvic symptoms, urinary incontinency with sexual activity, sexual avoidance, dyspareunia and decreased sexual arousal (Knoepp et al., 2010).

Interesting results were found through a couple of studies that used as method the projection of films monitoring different types of psychological states. One of these studies showed that sexually active women didn't have significant changes in cortisol, DHEA-S ratio and heart rate during the anxiety inducing film but at the same time had lower sexual arousal (Hamilton \& Meston, 2011). The other one, using a sample of young women with high and average stress that were sexually active, found that chronic daily stressors increase distraction and cortisol and increase sympathetic nervous system activity and DHEA-S (Hamilton \& Meston, 2013). In addition, one of these researchers using another method, expressive writing, not for measuring but for managing PTSD followed by sexual dysfunction problems, showed that by diminishing PTSD symptoms sexual dysfunction was ameliorated (Meston et al., 2013). Other findings on a sample of patients with psoriatic arthritis predicate that the dermatological symptoms of psoriasis are associated with anxiety, which is one of the main reasons that the prevalence of sexual dysfunction was higher among these patients, especially women (Kurizky \& Mota, 2012).

Stress was found to be an intermediate negative factor between sexual function and partner relationship in a sample of women with Type 2 Diabetes Mellitus. However, the association between partner relationship and female sexual function was proved a stronger factor than stress status (Shadman et al., 2014). A relevant finding about the importance of partner relationship was underlined on a study of 198 couples where the $34 \%$ of women reported hypoactive sexual desire and 13\% orgasmic problems (Bodenmann et al., 2010). In this study internal daily stress explains $9 \%$ to $26 \%$ of the variance of sexual problems and $47 \%$ of sexual aversion and sexual desire problems mainly explains the female sexual dysfunction except orgasmic problems and dyspareunia. External daily stress according to this study does not affect sexual dysfunction but affects relationship quality which is predictive for sexual arousal problems and vaginismus. Nonetheless, $68 \%$ do not pay attention to their sexual dysfunction because of their busy life conditions. As far as coping processes are concerned two studies revealed that emotional support, openness and positive coping strategies are followed by lower stress levels and are correlated with better orgasm and higher sexual satisfaction while introversion and negative coping strategies induce high distress (Crisp et al., 2013).

Sexual dysfunctions are of high prevalence on men granting serious effects on their quality of life. Premature ejaculation is likely the most prevalent type of sexual dysfunction with a percentage of $25 \%-40 \%$ global prevalence rate across all age groups (Park et al., 2010). Longer intravaginal ejaculatory latency time (IELT) led to reduced stress when respondents with less than 1 min IELT had the highest stress score especially on the category of partner's stress. Premature ejaculation causes high stress to the subjects and their partners and this relation was stronger for the younger (20s) (Park et al., 2010).

Another study examines the association between internal, external stress, critical life events and sexual dysfunction. From the above association internal stress and critical life events are strongly correlated with premature ejaculation, when in stressful conditions $10 \%$ of men reported premature ejaculation and $13 \%$ sexual desire problems. However, similarly to women, busy life conditions affect the attention that men pay on their sexual dysfunction at a percentage of 38\% (Bodenmann et al., 2010).

As cited previously, in a study conducted in patients with psoriatic arthritis was predicated that the dermatological symptoms of psoriasis are associated with anxiety, which is one of the main reasons for which the prevalence of sexual dysfunction was higher among these patients. A percentage of $50 \%$ of male patients, though, had erectile dysfunction problems (Kurizky \& Mota, 2012). Timed intercourse is a factor studied thoroughly on 
its impact on men's sexual function. In two studies investigating the relation among stress, timed intercourse and sexual dysfunction, men following a program of timed intercourse showed high percentages of sexual dysfunction (erectile, ejaculatory) (Byun et al., 2013). Men with delayed ejaculation had more stress which was mainly imposed by timed intercourse. Stress and anxiety are detrimental to erectile dysfunction frequency that is increased with the timed intercourse frequency. Obligatory coitus and compulsory sexual behavior lead to stress and anxiety and furthermore to sexual dysfunctions (Bak et al., 2012).

In the case of Veterans that suffer from PTSD, the relation among PTSD and sexual dysfunction is high (Breyer et al., 2014). Hosain et al. (2013) in a similar study found significant differences on the percentages of sexual dysfunctions related to PTSD. For the African Americans the relation was stronger compering to other races/ethnicities (Whites, Hispanics).

A study that was based on the review on current literature found that sexually dysfunctional individuals exhibit heightened levels of anxiety, suggesting an important role of anxiety in the subjective experience and maintenance of sexual disorders. Anxiety is considered to be the final pathway which leads to sexual dysfunction, while strong correlation evidence exists between erectile dysfunction and anxiety. Safe stress levels are found to increase sexuality, especially among men, while chronic levels of stress have detrimental effects on sexual function (McCabe et al., 2010).

\section{Conclusion}

The systematically reviewed literature proved the strong correlation among stress/anxiety and sexual functioning. The most studied sexual dysfunctions for men are erectile dysfunction and premature ejaculation, while for women are sexual desire/arousal and orgasmic disorders. Future studies are needed to investigate the pathways of how stress impacts on sexual dysfunctions. Finally, it would be of great interest for a systematic review to be done on the treatment and management of stress that leads to sexual dysfunction.

\section{References}

Bak, C. W., Lyu, S. W., et al. (2012). Erectile Dysfunction and Extramarital Sex induced by Timed Intercourse: A Prospective Study of 439 Men. Journal of Andrology, 33, 1245-1253. http://dx.doi.org/10.2164/jandrol.112.016667

Bodenmann, G., Atkins, D. C., \& Scaer, M. (2010). The Association between Daily Stress and Sexual Activity. Journal of Family Psychology, 24, 271-279. http://dx.doi.org/10.1037/a0019365

Breyer, B. N., Cohen, B. E., et al. (2014). Sexual Dysfunction in Male Iraq and Afghanistan War Veterans: Association with Posttraumatic Stress Disorder and other Combat-Related Mental Health Disorders: A Population-Based Cohort Study. The Journal of Sexual Medicine, 11, 75-83. http://dx.doi.org/10.1111/jsm.12201

Byun, J. S., Lyu, S. W., Seok, H. H., et al. (2013). Sexual Dysfunction Induced by Stress of Timed Intercourse and Medical Treatment. BJU International, 111, E227-E234. http://dx.doi.org/10.1111/j.1464-410X.2012.11577.x

Crisp, C. C., Vaccaro, C. M., et al. (2013). Is Female Sexual Dysfunction Related to Personality and Coping? An Exploratory Study. Sexual Medicine, 1, 69-75. http://dx.doi.org/10.1002/sm2.16

Hamilton, L. D., \& Meston, C. M. (2011). The Role of Salivary Cortisol and DHEA-S in Response to Sexual, Humorous, and Anxiety-Inducing Stimuli. Hormones and Behavior, 59, 765-771. http://dx.doi.org/10.1016/j.yhbeh.2010.12.011

Hamilton, L. D., \& Meston, C. M. (2013). Chronic Stress and Sexual Function in Women. The Journal of Sexual Medicine, 10, 2443-2454. http://dx.doi.org/10.1111/jsm.12249

Hosain, G. M., Latini, D. M., Kauth, M. R., Goltz, H. H., \& Helmer, D. A. (2013). Racial Differences in Sexual Dysfunction among Postdeployed Iraq and Afghanistan Veterans. American Journal of Men's Health, 5, 374-381. http://dx.doi.org/10.1177/1557988312471842

IsHak, W. W., \& Tobia, G. (2013). DSM-5 Changes in Diagnostic Criteria of Sexual Dysfunctions. Reproductive System \& Sexual Disorders, 2, 122.

Knoepp, L. R., et al. (2010). Sexual Complaints, Pelvic Floor Symptoms, and Sexual Distress in Women over Forty. The Journal of Sexual Medicine, 7, 3675-3682. http://dx.doi.org/10.1111/j.1743-6109.2010.01955.x

Kurizky, P. S., \& Mota, L. M. (2012). Sexual Dysfunction in Patients with Psoriasis and Psoriatic Arthritis—A Systematic Review. Revista Brasileira de Reumatologia, 52, 943-948. http://dx.doi.org/10.1590/S0482-50042012000600011

Lazarus, R. S., \& Folkman, S. (1984). Stress, Appraisal and Coping. New York: Springer.

McCabe, M., Althof, S. E., et al. (2010). Psychological and Interpersonal Dimensions of Sexual Function and Dysfunction. The Journal of Sexual Medicine, 7, 327-336. http://dx.doi.org/10.1111/j.1743-6109.2009.01618.x 
Meston, C. M., Lorenz, T. A., \& Stephenson, K. R. (2013). Effects of Expressive Writing on Sexual Dysfunction, Depression, and PTSD in Women with a History of Childhood Sexual Abuse: Results from a Randomized Clinical Trial. The Journal of Sexual Medicine, 10, 2177-2189. http://dx.doi.org/10.1111/jsm.12247

Park, H. J., Park, J. K., Park, K., et al. (2010). Prevalence of Premature Ejaculation in Young and Middle-Aged Men in Korea: A Multicenter Internet-Based Survey from the Korean Andrological Society. Asian Journal of Andrology, 12, 880-889. http://dx.doi.org/10.1038/aja.2010.9

Shadman, Z., Akhoundan, M., Poorsoltan, N., et al. (2014). Factors Associated with Sexual Function in Iranian Women with Type 2 Diabetes Mellitus: Partner Relationship as the Most Important Predictor. Iranian Red Crescent Medical Journal, 16, e14941. http://dx.doi.org/10.5812/ircmj.14941

Shindel, A. W., Breyer, B. N., \& Smith, J. F. (2013). Associations of Desire for Change in Sexual Life amongst Female Medical Students in North America. International Journal of Impotence Research, 25, 74-79. http://dx.doi.org/10.1038/ijir.2012.34

Shindel, A. W., Eisenberg, M. L., et al. (2011). Sexual Function and Depressive Symptoms among Female North American Medical Students. The Journal of Sexual Medicine, 8, 391-399. http://dx.doi.org/10.1111/j.1743-6109.2010.02085.x 